\title{
Transitioning Laser Ablation ICP-MS from Research Applications to Routine Trace Element Microanalysis: A Look at Quantitative Trace Element Analyses and Imaging
}

\author{
A.E. Koenig* \\ * United States Geological Survey, Laser Ablation ICP-MS Facility, MS973, Denver Federal \\ Center, \\ Denver, CO 80225
}

Trace element analyses at high spatial resolution shed light on processes that in some cases are just now beginning to be understood. Techniques for qualitative and quantitative trace element analyses at sub-millimeter scale resolutions include SIMS, EPMA, XRF (micro and synchrotron) and laser ablation ICP-MS. Each of these techniques has its own set of advantages and disadvantages. In support of work related to geological, biological and environmental problems our center has been researching methods for advancing quantitative analyses by EPMA and LA-ICP-MS. Trace element analyses by LA-ICP-MS has often been hampered by the lack of reference materials and validated methods. Here we review some of the previous work demonstrating the utility of LA-ICP-MS for trace elements in natural materials, new directions for routine analyses by LA-ICP-MS, results for matrices previously lacking reference materials or validated methods and an overview of quantitative trace element mapping by LA-ICP-MS.

The requirement of calibration reference materials to be of similar matrix to the target sample has been somewhat lessened since the development of new laser technologies such as $<200 \mathrm{~nm}$ wavelength lasers and femtosecond laser systems. However, numerous matrices exist where few materials of known concentration or sufficient homogeneity at $<100$ micrometer scales exist. The prevalence of LA-ICP-MS research in geological and mineralogical research has led to many of the reference materials that are currently available to be mostly geologically relevant. However, many sample matrices studied are either a matrix lacking a good reference material match or a matrix that may not have been validated with currently available reference materials. In many cases a matrix mismatched reference material may produce data fit for purpose, but lacking any secondary QC materials this validation is lacking. Further examination of recent developments of microanalytical reference materials developed by the USGS will be presented.

Methods validation for metallic sulfide and oxide minerals such as pyrite and magnetite will be presented using a range of calibration reference materials. Results for phosphatic material such as mineral apatite and biogenic apatite along with biogenic and mineral carbonate will be presented. In some cases the results contradict conventional wisdom on the degree of matrix matching required. In cases where external validation values are present it is possible to address which matrices work and when. External cross validation with EPMA values for minor elements as well as the use secondary reference materials as QC materials for trace elements provides information as to the performance in these cases. Some cases where LA-ICP-MS can produce wrong or incomplete results where methods are not carefully scrutinized will be presented. These possibly erroneous results are not presented as limitations of the technique in that they are only limitations if you are unaware of them or unable to develop methods that work. While further methods development for geological materials is important for geological research, the full adoption of LA-ICP-MS by the greater microanalytical 
community is still awaiting methods development for routine materials science applications, biological, and industrial materials. Results for some industrial materials such as metals, raw materials such as quartz powder, building materials such as concrete and biological samples such as bones and tissues will be presented. Comparison with EPMA and micro-XRF for some of these materials will be discussed.

Well known to the EPMA and micro-XRF community is the power of elemental mapping. Such imaging of where the elements "live" or are distributed in the sample provide information that is often crucial to the understanding of the sample. While much has been done to correct such images to be more quantitative these elemental maps are often subject to relative differences induced through sample or matrix effects and are not real differences. The same can be true for qualitative maps generated by raw ICP-MS response data when using LA-ICP-MS for imaging. Here we provide further methods development of quantitative trace element mapping using LA-ICP-MS for a range of samples looking specifically at ways to ensure that the concentrations are accurate within the elemental map. New methods for improving spatial resolution of trace element mapping by LAICP-MS will be presented. It is now possible to provide quantitative information on $<1 \mathrm{ppm}$ levels for some of the periodic table at $<10$ micron resolution. Laser ablation ICP-MS trace element imaging is faster than many complimentary techniques. Limitations still include the required ablation of the material providing some smearing in the depth component often on the scale of 10 micrometers or more. Imaging in chemically or mechanically diverse materials still represents a challenge for LA-ICP-MS. Some potential improvements on the methods for challenging applications will be discussed.

So is LA-ICP-MS ready to transition to more routine applications? I would say that the answer is yes. But the real question is "is the community ready to understand some of the requirements and challenges of the technique to face these routine applications?" The black box with to big "Go" button on the front that just spits out accurate and precise values for all samples and all trace elements does not quite exist yet. Much of the transition of many technologies such as LA-ICP-MS to the routine lab is not a limitation of the technique per se, but a real motivation of the community to take the steps necessary to use the technology for the applications appropriately. Hopefully this presentation will outline some of where the technique is at and where it is going for trace element analyses and imaging. 